\title{
Expression of Polymorphic Membrane Protein I of C. trachomatis
}

\author{
J.E. Goolsby,* P.M. Bavoil,** and R. Hsia* \\ *Core Imaging Facility, University of Maryland Dental School 650 West Baltimore Street, \\ Baltimore, MD 21201 \\ **Department of Microbial Pathogenesis, University of Maryland Dental School, 650 West \\ Baltimore Street, MD 21201
}

Chlamydia trachomatis is an obligate intracellular bacterium which causes genital and ocular infection in humans, with infertility and ectopic pregnancy among major sequelae in industrialized countries. The chlamydial developmental cycle includes two distinct forms, the extracellular, metabolically inert elementary body (EB) and the intracellular, metabolically active reticulate body (RB). A typical infection begins when an infectious EB attaches to and enters into the host cell. The EB then differentiates into a RB inside a specialized vacuole, the inclusion (Fig. 1). RBs multiply inside the inclusion and differentiate back into EBs 48 to 72 hours post infection. EBs are released into the environment by exocytosis or host cell lysis. Earlier studies have identified several surface proteins that play an important role in eliciting host responses or promoting attachment to the mucosal membrane and internalization [1]. Nine members of a gene family of polymorphic membrane proteins (Pmps), designated PmpA to I, have been identified in the C. trachomatis genome. Pmp proteins share similar repeat motifs, a signal sequence and are structurally similar to autotransporter proteins identified in other bacteria [2].

Serologic studies have revealed that Pmp proteins are immunogenic and that different patients may exhibit variable antibody responses to different Pmp Proteins [3]. Pmp-specific immunofluorescence (IF) labeling in C. trachomatis-infected Hela cells has shown that Pmp protein expression varies among individual inclusions (Fig. 2). It is suggested that Pmp protein polymorphism may be a mechanism of antigenic variation that Chlamydia use to evade the host immune response. Although all pmp genes are transcribed, not all Pmp proteins have been consistently detected by western blot or IF. While some studies have revealed that all Pmp proteins may be surface exposed in EBs [3], other have suggested that Pmp proteins are differentially located in EBs and RBs [4].

Here, we perform immunogold labeling of EBs in C. trachomatis-infected Hela cells to identify the location of Pmp proteins. C. trachomatis-infected Hela cells were embedded in unicryl at $-20^{\circ} \mathrm{C}$ by using "progressive lowering of temperature" (PLT) technique. Ultrathin sections of embedded cells were labeled with anti-PmpI antibody to investigate localization and possible interactions. Our results are consistent with previous IF data that Pmp expression may vary among individual chlamydiae in the same inclusion. Interestingly, EBs appeared to exhibit more PmpI labeling than RBs. PmpI labeling was predominantly intracellular in EBs and membrane-associated in RBs. Furthermore, not all chlamydiae in the same inclusion exhibited PmpI labeling. Possible interpretations are that PmpI-negative labeling may result from a polar distribution of the label or from the switching off of $p m p I$ expression. In summary, our results suggest that Pmp proteins may be differentially expressed and located during chlamydial development. 
[1] P.M. Bavoil. et al., Microbiology. 136 (2000) 2723.

[2] K.A. Swanson et al., Infect. Immun. 77 (2009) 508.

[3] C. Tan et al., Infect. Immun. 77 (2009) 3218.

[4] A.O. Kiselev et al., PLoS ONE. 2 (2007). e568.
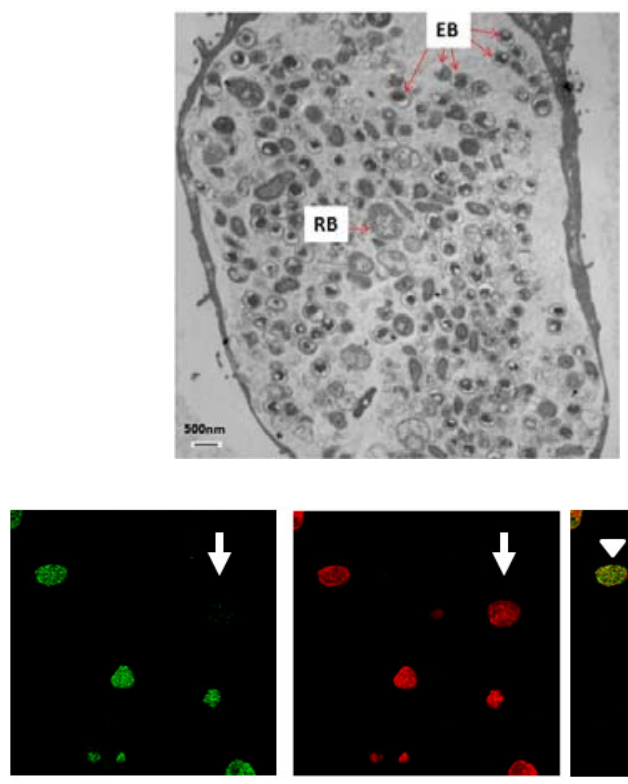

$\alpha P m p G$

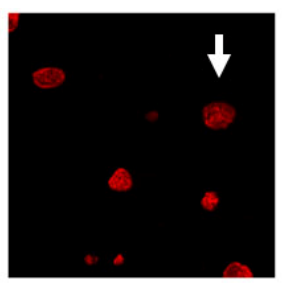

$\alpha E F-t u$
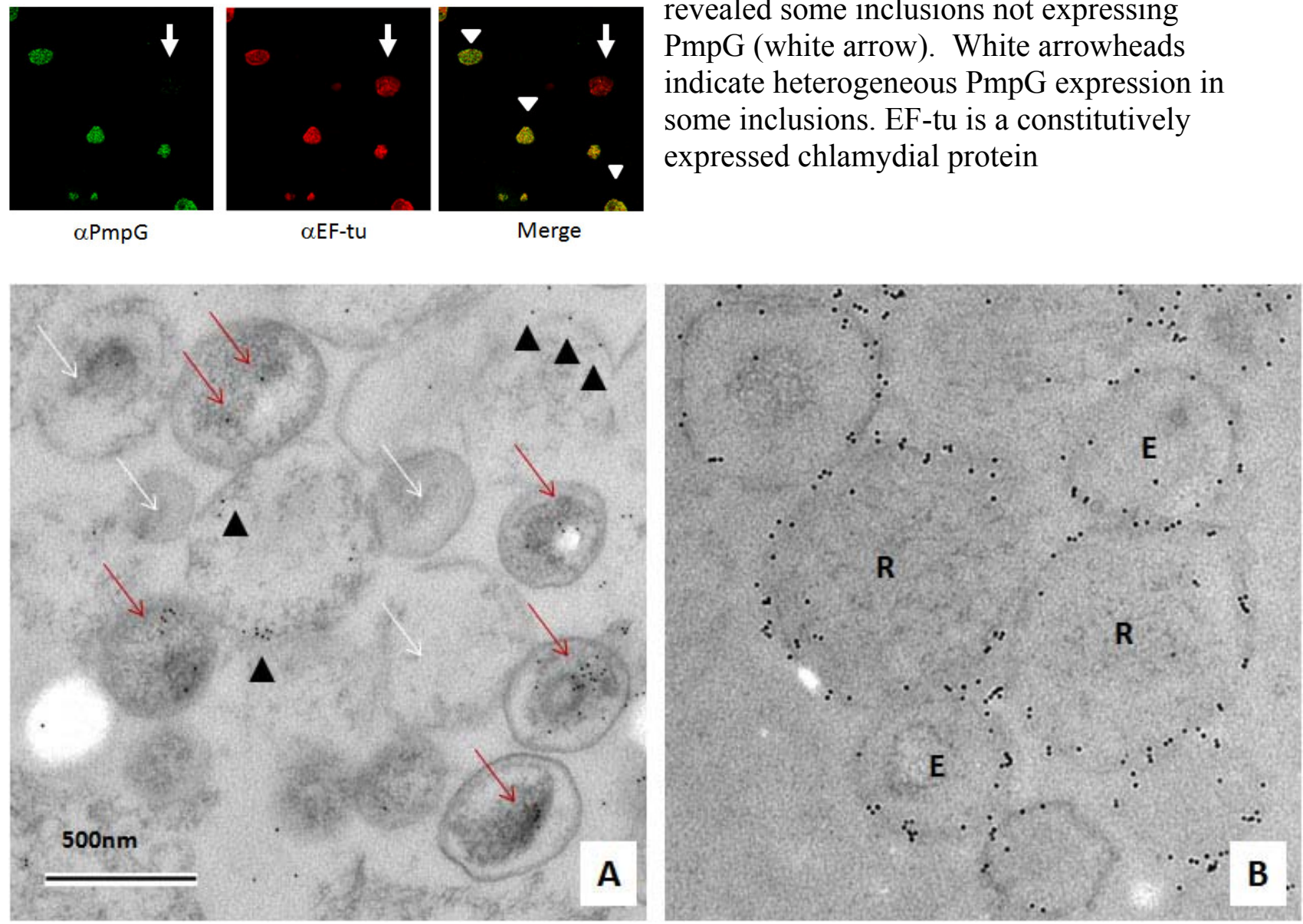

FIG. 2. IFA labeling of chlamydial inclusions revealed some inclusions not expressing PmpG (white arrow). White arrowheads indicate heterogeneous PmpG expression in some inclusions. EF-tu is a constitutively expressed chlamydial protein

FIG. 1. A late stage $C$. trachomatis inclusion is crowded with mostly EBs and some RBs . EBs are characterized by an electron dense core and are smaller.

FIG. 3. Immunogold labeling with anti PmpI (A) and anti MOMP (B) antibodies of inclusions within infected Hela cells $40 \mathrm{hr}$ post-infection. Red arrows indicate intracellular labeling of PmpI in EBs. White arrows indicate chlamydiae that do not have PmpI labeling. Black arrowheads indicate membrane-associated PmpI labeling in RBs. MOMP is the chlamydial major outer membrane protein which serves as a positive control. E: EB; R: RB. This figure is representative of more than three labeling experiments. 